\title{
A!
}

This is an electronic reprint of the original article.

This reprint may differ from the original in pagination and typographic detail.

Ollikainen, T.; Masuda, S.; Möttönen, M.; Nakahara, M.

\section{Counterdiabatic vortex pump in spinor Bose-Einstein condensates}

Published in:

Physical Review A

DOI:

10.1103/PhysRevA.95.013615

Published: 17/01/2017

Document Version

Publisher's PDF, also known as Version of record

Please cite the original version:

Ollikainen, T., Masuda, S., Möttönen, M., \& Nakahara, M. (2017). Counterdiabatic vortex pump in spinor Bose-

Einstein condensates. Physical Review A, 95(1), 1-7. [013615]. https://doi.org/10.1103/PhysRevA.95.013615

This material is protected by copyright and other intellectual property rights, and duplication or sale of all or part of any of the repository collections is not permitted, except that material may be duplicated by you for your research use or educational purposes in electronic or print form. You must obtain permission for any other use. Electronic or print copies may not be offered, whether for sale or otherwise to anyone who is not an authorised user. 


\title{
Counterdiabatic vortex pump in spinor Bose-Einstein condensates
}

\author{
T. Ollikainen, ${ }^{1}$ S. Masuda, ${ }^{1}$ M. Möttönen, ${ }^{1,2}$ and M. Nakahara ${ }^{1,3}$ \\ ${ }^{1}$ QCD Labs, COMP Centre of Excellence, Department of Applied Physics, Aalto University, P.O. Box 13500, FI-00076 Aalto, Finland \\ ${ }^{2}$ University of Jyväskylä, Department of Mathematical Information Technology, P.O. Box 35, FI-40014 University of Jyväskylä, Finland \\ ${ }^{3}$ Research Center for Quantum Computing and Department of Physics, Kinki University, Higashi-Osaka, 577-8502, Japan
}

(Received 14 October 2016; published 17 January 2017)

\begin{abstract}
Topological phase imprinting is a well-established technique for deterministic vortex creation in spinor BoseEinstein condensates of alkali-metal atoms. It was recently shown that counterdiabatic quantum control may accelerate vortex creation in comparison to the standard adiabatic protocol and suppress the atom loss due to nonadiabatic transitions. Here we apply this technique, assisted by an optical plug, for vortex pumping to theoretically show that sequential phase imprinting up to 20 cycles generates a vortex with a very large winding number. Our method significantly increases the fidelity of the pump for rapid pumping compared to the case without the counterdiabatic control, leading to the highest angular momentum per particle reported to date for the vortex pump. Our studies are based on numerical integration of the three-dimensional multicomponent Gross-Pitaevskii equation, which conveniently yields the density profiles, phase profiles, angular momentum, and other physically important quantities of the spin- 1 system. Our results motivate the experimental realization of the vortex pump and studies of the rich physics it involves.
\end{abstract}

DOI: 10.1103/PhysRevA.95.013615

\section{INTRODUCTION}

When the Bose-Einstein condensate (BEC) of alkali-metal atoms was discovered in 1995 [1,2], some of the immediate issues to be clarified were whether this system exhibits quantum coherence and whether it shows superfluidity, similar to that of superfluid ${ }^{4} \mathrm{He}$. Quantized vortices are manifestations of these properties and their realization has been one of the main research topics of experimental BEC physics since the discovery of the atomic BECs [3]. In general, the existence of stable vortices and other topological objects is attributed to an order parameter manifold with nontrivial homotopy groups supporting these objects $[4,5]$.

Methods to create vortices in BECs by so-called topological phase imprinting have been proposed in Refs. [6-12] and these proposals were later experimentally realized by several groups [13-19]. In addition to vortex creation, an extension of this method into a nontrivially three-dimensional scenario [20] has been used in the experimental creation of a Dirac monopole in the synthetic magnetic field of a spinor BEC [21]. Other methods to create vortices include the utilization of moving laser beams [22-24], rotating trap potentials [25], LaguerreGauss beams [26], and merging multiple condensates [27]. The advantages of the topological phase imprinting method are that it creates vortices deterministically at a desired location and that almost all atoms in the condensate acquire the desired angular momentum.

The operating principle of a vortex pump [28] is based on sequential application of the topological phase imprinting protocol, thus increasing the winding number by $2 F$ for each vortex pumping cycle, where $F$ is the quantum number of the hyperfine spin. Conventionally, various vortex pumping methods resort to adiabatic control of the system to imprint local Berry phase [29-31]. Hence, rapid pumping gives rise to errors owing to unwanted nonadiabatic transitions, eventually leading to the degradation of the pump. On the other hand, a vortex with a large winding number is dynamically unstable into splitting into multiple single-quantum vortices [32], motivating faster vortex pumping. The stability of the large-winding-number states has been studied [33,34], as well as their splitting dynamics [35].

Importantly, the counterdiabatic (CD) protocol [36,37], which is sometimes referred to as assisted adiabatic population transfer or shortcut to adiabaticity [38,39], can generate the same state as the corresponding adiabatic dynamics in shorter time. Therefore, it can be utilized to overcome several problems in adiabatic quantum control, for example, in cases in which the population transfer efficiency is limited by decoherence, three-body losses, and external noise in the control parameters of, e.g., isolated atoms and molecules [36,40,41], spin chain systems [42,43], Bose-Einstein condensates [44,45], and electron spin of a single nitrogen-vacancy center in a diamond [46]. Very recently, the CD protocol was also found to speed up the topological phase imprinting method [47]. However, vortex pumping using the $\mathrm{CD}$ protocol has not been reported to date.

The purpose of this paper is to demonstrate that the CD quantum control, assisted by an optical plug, can accelerate the vortex pump, and hence create a large-winding-number vortex in a short time. This also serves to reduce the atom loss from the trap. We consider a spin-1 BEC consisting of ${ }^{87} \mathrm{Rb}$ atoms in an optical trap with a three-dimensional quadrupole field present. The parameters in the simulations are set to experimentally feasible values according to Refs. [21,48]. In addition, we introduce an optical plug along the symmetry axis of the condensate to prevent transitions between the hyperfine spin states during the fast magnetic field ramp. We study the fidelity of the vortex creation and the state of the condensate for up to 20 vortex pumping cycles by numerically integrating the three-dimensional multicomponent Gross-Pitaevskii equation.

This paper is organized as follows. In Sec. II, we analyze single vortex-creation ramps with the $\mathrm{CD}$ quantum control. We consider four cases: linear and nonlinear ramps with and without the $\mathrm{CD}$ field. We compare the performance of these schemes to show that the nonlinear ramp with the CD protocol assisted by an optical plug yields the highest fidelity. Furthermore, the effect of the condensate aspect ratio on the 
vortex creation fidelity is studied. In Sec. III, we apply the results obtained in Sec. II to vortex pumping. Detailed density and phase profiles are studied for nonlinear ramps with the CD field. Section IV is devoted to conclusions.

\section{TOPOLOGICAL VORTEX IMPRINTING WITH COUNTERDIABATIC FIELD}

\section{A. Mean-field theory and topological vortex imprinting}

The mean-field order parameter of the spin-1 BEC is represented in the basis of the $z$-quantized spin states, $\quad\{|+1\rangle,|0\rangle,|-1\rangle\}$, as $\Psi(\boldsymbol{r}, t)=\left(\psi_{+1}(\boldsymbol{r}, t), \psi_{0}(\boldsymbol{r}, t)\right.$, $\left.\psi_{-1}(\boldsymbol{r}, t)\right)_{z}^{T}$, where the subscript in each spinor component denotes the magnetic quantum number along $z$. Furthermore, we write $\psi_{k}(\boldsymbol{r}, t)=\sqrt{n_{k}(\boldsymbol{r}, t)} \exp \left[i \phi_{k}(\boldsymbol{r}, t)\right]$, where $n_{k}(\boldsymbol{r}, t)$ is the particle density and $\phi_{k}(\boldsymbol{r}, t)$ is the phase of the spinor component $k$. The dynamics of the mean-field order parameter are solved in three dimensions employing the Gross-Pitaevskii (GP) equation

$$
\begin{aligned}
i \hbar \partial_{t} \Psi(\boldsymbol{r}, t)= & {\left[-\frac{\hbar^{2}}{2 m} \nabla^{2}+V(\boldsymbol{r})+c_{0} \Psi(\boldsymbol{r}, t)^{\dagger} \Psi(\boldsymbol{r}, t)\right.} \\
& \left.+c_{2} \Psi(\boldsymbol{r}, t)^{\dagger} \boldsymbol{F} \Psi(\boldsymbol{r}, t) \cdot \boldsymbol{F}+g_{F} \mu_{\mathrm{B}} \boldsymbol{B}(\boldsymbol{r}, t) \cdot \boldsymbol{F}\right] \\
& \times \Psi(\boldsymbol{r}, t),
\end{aligned}
$$

where the external optical potential is given by $V(\boldsymbol{r})=$ $V_{\text {opt }}(\rho, z)+V_{\text {plug }}(\rho)$, the harmonic part is $V_{\text {opt }}(\rho, z)=$ $m\left(\omega_{\rho}^{2} \rho^{2}+\omega_{z}^{2} z^{2}\right) / 2$, and $V_{\text {plug }}(\rho)=A_{\text {plug }} \exp \left(-\rho^{2} / \rho_{\text {plug }}^{2}\right)$ is the optical plug potential defined in the cylindrical coordinate system $(\rho, \varphi, z)$. Furthermore, $\boldsymbol{B}(\boldsymbol{r}, t)$ is the external timedependent magnetic field, and $\boldsymbol{F}=\left(F_{x}, F_{y}, F_{z}\right)$ is a vector composed of the standard dimensionless spin- 1 matrices. The constants $c_{0}=4 \pi \hbar^{2}\left(a_{0}+2 a_{2}\right) /(3 m)$ and $c_{2}=4 \pi \hbar^{2}\left(a_{2}-\right.$ $\left.a_{0}\right) /(3 m)$ are the coupling constants related to the densitydensity and spin-spin interactions [49,50], respectively, $g_{F}$ is the Landé $g$ factor, and $\mu_{\mathrm{B}}$ is the Bohr magneton. For $F=1{ }^{87} \mathrm{Rb}$ atoms, the $s$-wave scattering lengths are given by $a_{0}=5.387 \mathrm{~nm}$ and $a_{2}=5.313 \mathrm{~nm}$ [51], the atomic mass by $m=1.443 \times 10^{-25} \mathrm{~kg}$, and $g_{F}=-1 / 2$. The number of atoms is set to $N=2.1 \times 10^{5}$ throughout the simulations and the optical plug parameters are set to $\rho_{\text {plug }}=1.80 \mu \mathrm{m}$ and $A_{\text {plug }}=6.6 \times 10^{-30} \mathrm{~J}$ in the cases in which the optical plug is employed.

In the topological vortex imprinting scheme considered, we employ an external magnetic field consisting of a threedimensional quadrupole field with an additional bias field along $z$ defined as

$$
\begin{aligned}
\boldsymbol{B}(\boldsymbol{r}, t) & =b_{\mathrm{q}}(x \hat{\boldsymbol{x}}+y \hat{\boldsymbol{y}}-2 z \hat{\boldsymbol{z}})+B_{0}(t) \hat{\boldsymbol{z}} \\
& =b_{\mathrm{q}}(\rho \cos \varphi \hat{\boldsymbol{x}}+\rho \sin \varphi \hat{\boldsymbol{y}})+B_{z}(z, t) \hat{\boldsymbol{z}},
\end{aligned}
$$

where $b_{\mathrm{q}}$ is the strength of the gradient field and $B_{0}(t)$ is the bias field component along $z$. We have further defined $B_{z}(z, t)=B_{0}(t)-2 b_{\mathrm{q}} z$

Let us consider an atom at a point $\boldsymbol{r}$ at time $t$. In the presence of the magnetic field $\boldsymbol{B}$, considering only the Zeeman term in the Hamiltonian, $\mathcal{H}_{\mathrm{Z}}=g_{F} \mu_{\mathrm{B}} \boldsymbol{B} \cdot \boldsymbol{F}$, we have three eigenstates corresponding to the weak-field-seeking state (WFSS), which has the highest energy, the neutral state (NS) with zero energy, and the strong-field-seeking state (SFSS), which has the lowest energy. In the $z$-quantized basis, these eigenstates are represented as

$$
\begin{array}{r}
|\mathrm{WFSS}\rangle \hat{=} \frac{1}{2 B}\left(\begin{array}{c}
B-B_{z} \\
-\sqrt{2} b_{\mathrm{q}} \rho e^{i \varphi} \\
\left(B+B_{z}\right) e^{2 i \varphi}
\end{array}\right)_{z}, \\
|\mathrm{NS}\rangle \hat{=} \frac{1}{\sqrt{2} B}\left(\begin{array}{c}
-b_{\mathrm{q}} \rho \\
\sqrt{2} B_{z} e^{i \varphi} \\
b_{\mathrm{q}} \rho e^{2 i \varphi}
\end{array}\right)_{z} \\
|\mathrm{SFSS}\rangle \hat{=} \frac{1}{2 B}\left(\begin{array}{c}
B+B_{z} \\
\sqrt{2} b_{\mathrm{q}} \rho e^{i \varphi} \\
\left(B-B_{z}\right) e^{2 i \varphi}
\end{array}\right)_{z}
\end{array}
$$

where $B(\rho, z, t)=\sqrt{b_{\mathrm{q}}^{2} \rho^{2}+B_{z}(z, t)^{2}}$ is the total magneticfield strength. In contrast to the earlier work in Ref. [47], here we consider an optically trapped condensate and the magnetic field is primarily used only to control the spin state. Hence we are free to choose also the magnetically untrapped SFSS as the initial state. Under a strong bias field we have $B \approx B_{z}$ at the location of the atoms, and consequently the state is approximately $(1,0,0)_{z}^{T}$. As the bias field is adiabatically ramped to a large negative value, such that $B_{z} \approx-B$, the state transforms into approximately $\left(0,0, e^{2 i \varphi}\right)_{z}^{T}$. The appearance of the azimuthal dependence in the phase factor can be attributed to the accumulation of the Berry phase during the adiabatic change of the bias field [52].

While the bias field is inverted, there appears a space-time point where the magnetic field and hence Zeeman energy gap between the eigenstates vanishes. Near this point, transitions from SFSS to NS and WFSS take place. However, atoms in the SFSS are naturally repelled from the location of the magnetic-field zero, which suppresses such transitions. Due to this reason, we take SFSS as the initial state in most of our simulations. Additionally, we employ the optical plug in some of the simulations to further diminish the atom loss and to stabilize the resulting multiquantum vortex [33].

\section{B. Counterdiabatic field for a three-dimensional quadrupole field}

In an adiabatic process, the populations in the instantaneous eigenstates of the time-dependent Hamiltonian remain constant. If the system is not driven slowly enough, transitions between the quantum states take place. Counterdiabatic protocol can be used to design an auxiliary term in the Hamiltonian to overcome the requirement of slow driving. This auxiliary term, in combination with the original Hamiltonian, generates the same final state as the adiabatic process [36,39].

The dynamics of a spin-1 system in the presence of a changing magnetic field are described by the Zeeman Hamiltonian $\mathcal{H}_{\mathrm{Z}}$. The auxiliary Hamiltonian provided by the CD scheme in this case is given by $\mathcal{H}_{\mathrm{CD}}=g_{F} \mu_{\mathrm{B}} \boldsymbol{B}_{\mathrm{CD}} \cdot \boldsymbol{F}$, where the so-called CD field reads $\boldsymbol{B}_{\mathrm{CD}}=\hbar \boldsymbol{B} \times \partial_{t} \boldsymbol{B} /\left(g_{F} \mu_{\mathrm{B}}|\boldsymbol{B}|^{2}\right)$ [37].

In the following, we may approximate $B_{z}(z, t) \approx B_{z}(0, t)=$ $B_{0}(t)$. Thus the CD field in the topological vortex imprinting 
method, according to protocol in Eq. (2), assumes the form

$$
\boldsymbol{B}_{\mathrm{CD}}(\boldsymbol{r}, t)=\frac{\hbar \dot{B}_{0}(t) b_{\mathrm{q}}}{g_{F} \mu_{\mathrm{B}} B^{2}(\rho, 0, t)}(y \hat{\boldsymbol{x}}-x \hat{\boldsymbol{y}}),
$$

where $\dot{B}_{0}(t)$ denotes the temporal derivative of the bias field. Hence the required external magnetic field including the $\mathrm{CD}$ field is thus $\boldsymbol{B}+\boldsymbol{B}_{\mathrm{CD}}$. It is also possible to solve the CD field without setting $z=0$, but it turns out to be of complicated form and not likely conveniently realizable with the current experimental equipment. Furthermore, we make the approximation $\rho=\rho_{0}$ in the denominator of Eq. (6) due to the requirement $\nabla \cdot\left(\boldsymbol{B}+\boldsymbol{B}_{\mathrm{CD}}\right)=0$ imposed by Maxwell's equations.

Implementing the modified magnetic field $\boldsymbol{B}+\boldsymbol{B}_{\mathrm{CD}}$ is experimentally challenging, since it would require two separate sets of exactly aligned quadrupole coils. In order to make this scheme experimentally convenient, we consider a time-dependent unitary transformation $U(t)=$ $\exp \left[-i \alpha(t) F_{z}\right]$, similar to that in Ref. [47], which introduces a position-independent rotation of an angle

$$
\alpha(t)=\arctan \left(\frac{\left|\boldsymbol{B}_{\mathrm{CD}}(\boldsymbol{r}, t)\right|}{b_{\mathrm{q}} \rho}\right)
$$

about the $z$ axis. The order parameter transforms into $\Psi^{\prime}(\boldsymbol{r}, t)=U(t) \Psi(\boldsymbol{r}, t)$. In the beginning and at the end of the vortex creation process of duration $T$, we have $|\boldsymbol{B}| \gg\left|\boldsymbol{B}_{\mathrm{CD}}\right|$, and consequently $\alpha(0)=\alpha(T) \approx 0$, i.e., $U(0)=U(T) \approx I$, where $I$ is the identity. Hence the order parameters $\Psi^{\prime}(\boldsymbol{r}, t)$ and $\Psi(\boldsymbol{r}, t)$ coincide in the beginning and at the end of the ramp, and we may steer the system with the effective Hamiltonian for $\Psi^{\prime}(\boldsymbol{r}, t)$ to achieve the desired CD dynamics.

The Zeeman part of the Hamiltonian (1) transforms for $\Psi^{\prime}(\boldsymbol{r}, t)$ into $g_{F} \mu_{\mathrm{B}} U(t)\left(\boldsymbol{B}+\boldsymbol{B}_{\mathrm{CD}}\right) \cdot \boldsymbol{F} U(t)^{\dagger}$. This gives rise to a rotation of the magnetic field by an angle $\alpha(t)$ about the $z$ axis. Furthermore, the transformed Hamiltonian includes an additional term $-i \hbar U(t) \partial_{t} U(t)^{\dagger}=\hbar \dot{\alpha}(t) F_{z}$, which we take into consideration by adding a magnetic field $\hbar \dot{\alpha}(t) /\left(g_{F} \mu_{\mathrm{B}}\right)$ along $z$. The resulting magnetic field, which we use in the simulations, assumes the form

$$
\begin{aligned}
\tilde{\boldsymbol{B}}(\boldsymbol{r}, t)= & b_{\mathrm{q}} \rho \sqrt{1+\left[\frac{\hbar \dot{B}_{0}(t)}{g_{F} \mu_{\mathrm{B}} B^{2}\left(\rho_{0}, 0, t\right)}\right]^{2}}(\hat{\boldsymbol{x}}+\hat{\boldsymbol{y}}-2 \hat{z}) \\
& +\left[B_{0}(t)+\frac{\hbar}{g_{F} \mu_{\mathrm{B}}} \dot{\alpha}(t)\right] \hat{z} .
\end{aligned}
$$

As is evident from the above equation, also the gradient field along $z$ is affected by the CD field in our simulations. As a result, only one set of quadrupole coils is required to implement the resulting magnetic field.

In the vortex creation scheme employed here, the bias field $B_{z}$ is ramped from a large positive value to a large negative value while keeping the quadrupole field strength $b_{\mathrm{q}}$ constant. We consider two different ramping functions

$$
B_{0}^{1}(t)=(1-2 t / T) B_{\mathrm{i}}
$$

and

$$
B_{0}^{\mathrm{nl}}(t)=g(t)(1-2 t / T) B_{\mathrm{i}},
$$
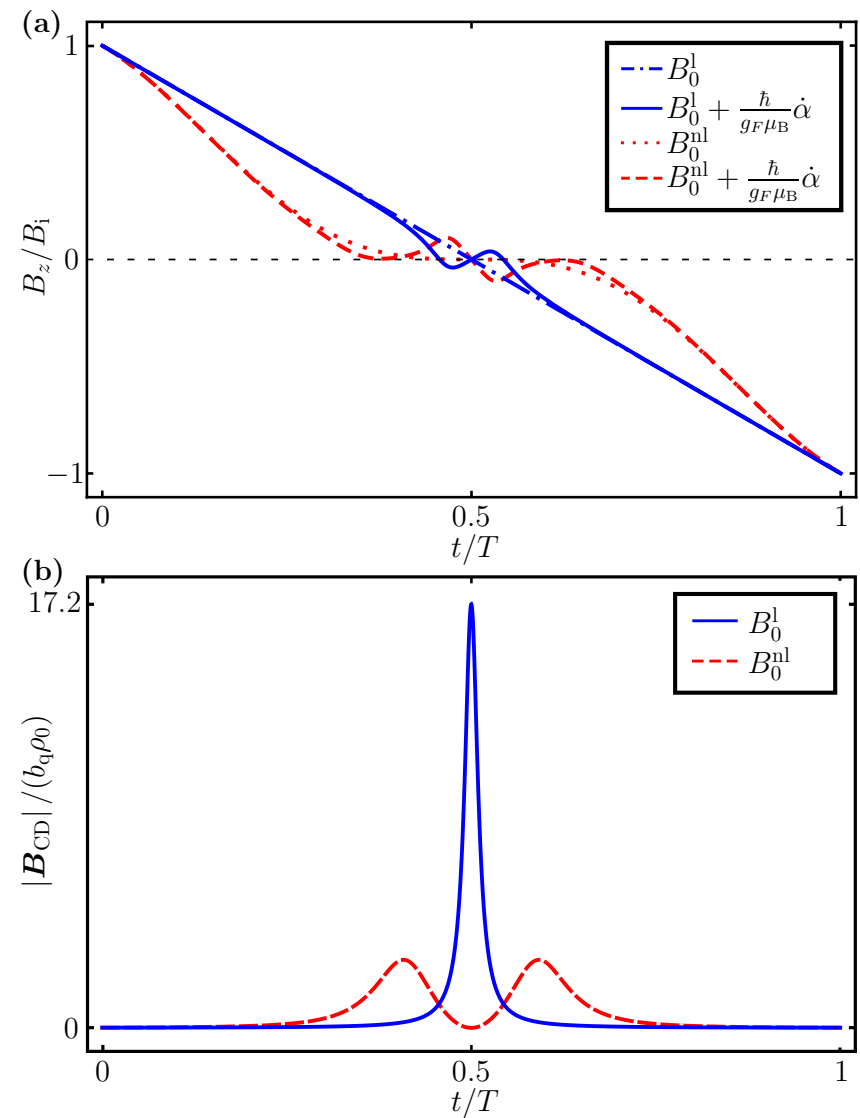

FIG. 1. (a) Bias field component along $z$ and (b) the strength of the CD field as functions of time $t$. We choose $B_{\mathrm{i}}=0.5 \mathrm{G}, \rho_{0}=$ $3 \mu \mathrm{m}, b_{\mathrm{q}}=3.675 \mathrm{G} / \mathrm{cm}$, and the total ramp time $T=1.16 \mathrm{~ms}$. In (a), the dash-dotted blue and the dotted red lines correspond to the linear and nonlinear ramps, respectively. The solid blue and the dashed red lines correspond to the linear and nonlinear ramps, respectively, with the term $\hbar \dot{\alpha} /\left(g_{F} \mu_{\mathrm{B}}\right)$ taken into account. In (b), the solid blue and the dashed red lines correspond to the linear and nonlinear ramps, respectively.

where $T$ is the duration of the ramp, $t \in[0, T]$, and $B_{\mathrm{i}}$ is the initial strength of the magnetic field. Ramping functions $B_{0}^{1}$ and $B_{0}^{\mathrm{nl}}$ are henceforth referred to as linear and nonlinear ramping functions, respectively. For the nonlinear ramp, we set

$$
g(t)=\frac{1}{2}\left\{1-\cos \left[\frac{2 \pi(t-T / 2)}{T}\right]\right\} .
$$

The ramp functions $B_{0}^{1}$ and $B_{0}^{\mathrm{nl}}$, their transformed counterparts, and the strength of the CD field for both ramp functions are shown in Fig. 1. The nonlinear ramp requires a weaker CD field strength compared with the linear ramp, hence reducing the amount of electric current needed in the quadrupole coils.

\section{Fidelity of vortex creation using counterdiabatic quantum control}

We define the fidelity of vortex creation as the fraction of atoms trapped in the SFSS, $N_{\mathrm{SFSS}}(t)$, with respect to the conserved total atom number, $N$, after a single creation ramp. Initially all atoms reside in the SFSS, i.e., $N_{\mathrm{SFSS}}(0)=N$. 
In an ideal case, without any nonadiabatic excitations, we would have $N_{\mathrm{SFSS}}(T)=N$. The initial and final values for the bias field are set to $B_{0}(0)=0.5 \mathrm{G}$ and $B_{0}(T)=-0.5 \mathrm{G}$, respectively. The quadrupole field strength is linearly ramped on in the beginning of the simulations and off at the end of the simulations with the bias field kept constant. The ramp times for setting the quadrupole field on and off are both roughly $0.06 \mathrm{~ms}$. Hence we can evaluate $N_{\mathrm{SFSS}}=$ $\int d \boldsymbol{r}\left|\psi_{+1}\right|^{2}$ at the beginning and $N_{\mathrm{SFSS}}=\int d \boldsymbol{r}\left|\psi_{-1}\right|^{2}$ at the end of the simulations. We consider two cases for the
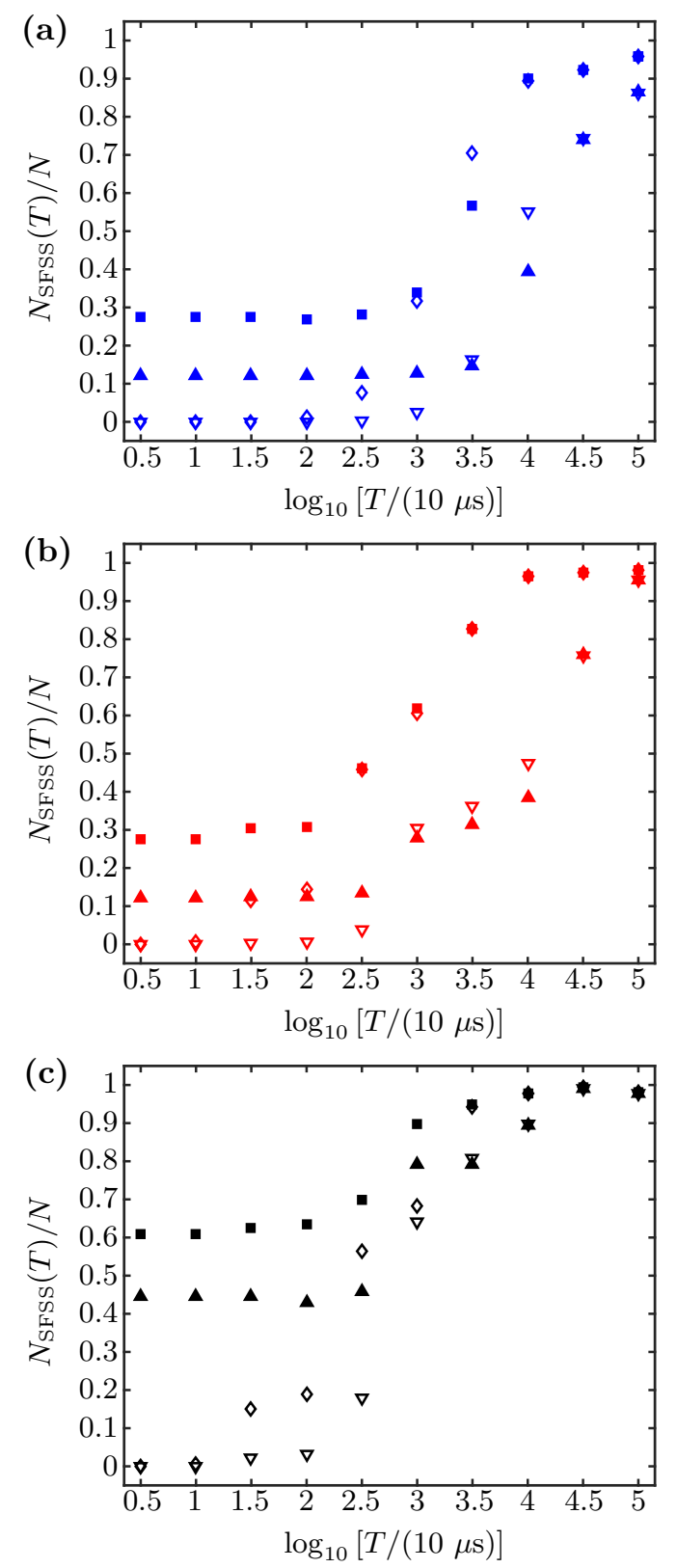

FIG. 2. Fidelity of the vortex creation as a function of the ramp time. We study (a) linear ramps, (b) nonlinear ramps, and (c) nonlinear ramps with the optical plug. Filled squares and empty diamonds correspond to the case with and without the CD field, respectively, for $\left(\omega_{\rho}, \omega_{z}\right)=2 \pi \times(24.8,124) \mathrm{Hz}$, and filled upward-pointing and empty downward-pointing triangles correspond to the case with and without the CD field, respectively, for $\left(\omega_{\rho}, \omega_{z}\right)=2 \pi \times(124,164) \mathrm{Hz}$. optical trapping potentials: $\left(\omega_{\rho}, \omega_{z}\right)=2 \pi \times(124,164) \mathrm{Hz}$ and $\left(\omega_{\rho}, \omega_{z}\right)=2 \pi \times(24.8,124) \mathrm{Hz}$. These correspond to slightly oblate and moderately oblate three-dimensional condensates, respectively. We choose the parameter $\rho_{0}$ to approximately correspond to the radial coordinate of the density maximum of the condensate at the beginning of the simulations: for slightly (moderately) oblate condensates we set $3.0(6.7) \mu \mathrm{m}$ in the case without the plug and $6.0(13.4) \mu \mathrm{m}$ with the plug. The optimal value for $\rho_{0}$ depends on the ramp time $T$ [47], and it is not fully optimized for all ramp times.

The resulting fidelity of the vortex creation is presented in Fig. 2 as a function of the ramp time $T$. The effect of the $\mathrm{CD}$ field is most dramatic for brief ramps, i.e., when the bias field is controlled in a nonadiabatic manner. For $T=10 \mu \mathrm{s}$, both linear and nonlinear ramps without the optical plug give essentially the same result: the CD field improves the fidelity from almost zero to 0.1 and 0.3 for slightly and moderately oblate condensates, respectively. At longer times, $T>10 \mu \mathrm{s}$, nonlinear ramp yields slightly higher fidelity since the zero of the field moves more slowly in the condensate region.

The optical plug further enhances the fidelity for all ramp times, since the atoms are repelled from the path of the field zero. Indeed, the fidelity is very close to unity at $T>100 \mathrm{~ms}$ if the optical plug is employed, regardless whether the CD
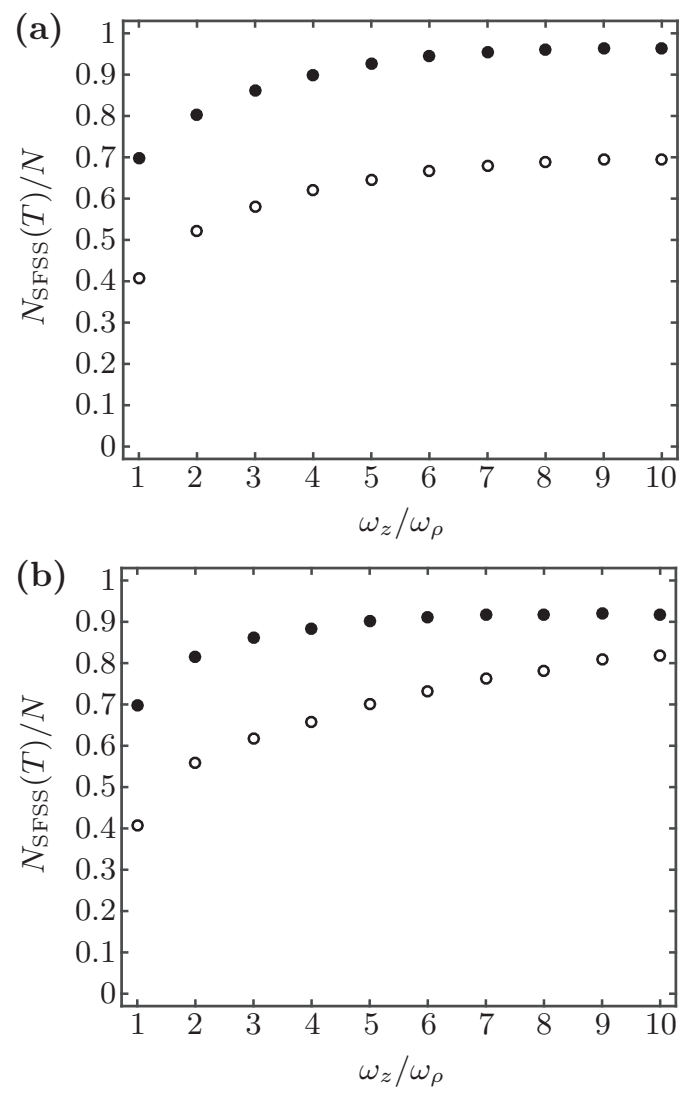

FIG. 3. Fidelity of the vortex creation as a function of the aspect ratio of the trap frequencies $\omega_{z} / \omega_{\rho}$ in the case of nonlinear ramps and optical plug, for (a) $\omega_{\rho}=2 \pi \times 124 \mathrm{~Hz}$ and for (b) $\omega_{z}=2 \pi \times$ $124 \mathrm{~Hz}$. Filled and empty circles correspond to the cases with and without the CD field, respectively. The ramp time $T=5.78 \mathrm{~ms}$ and the CD parameter $\rho_{0}=\sqrt{(2 \pi \times 124 \mathrm{~Hz}) / \omega_{\rho}} \times 6 \mu \mathrm{m}$. 

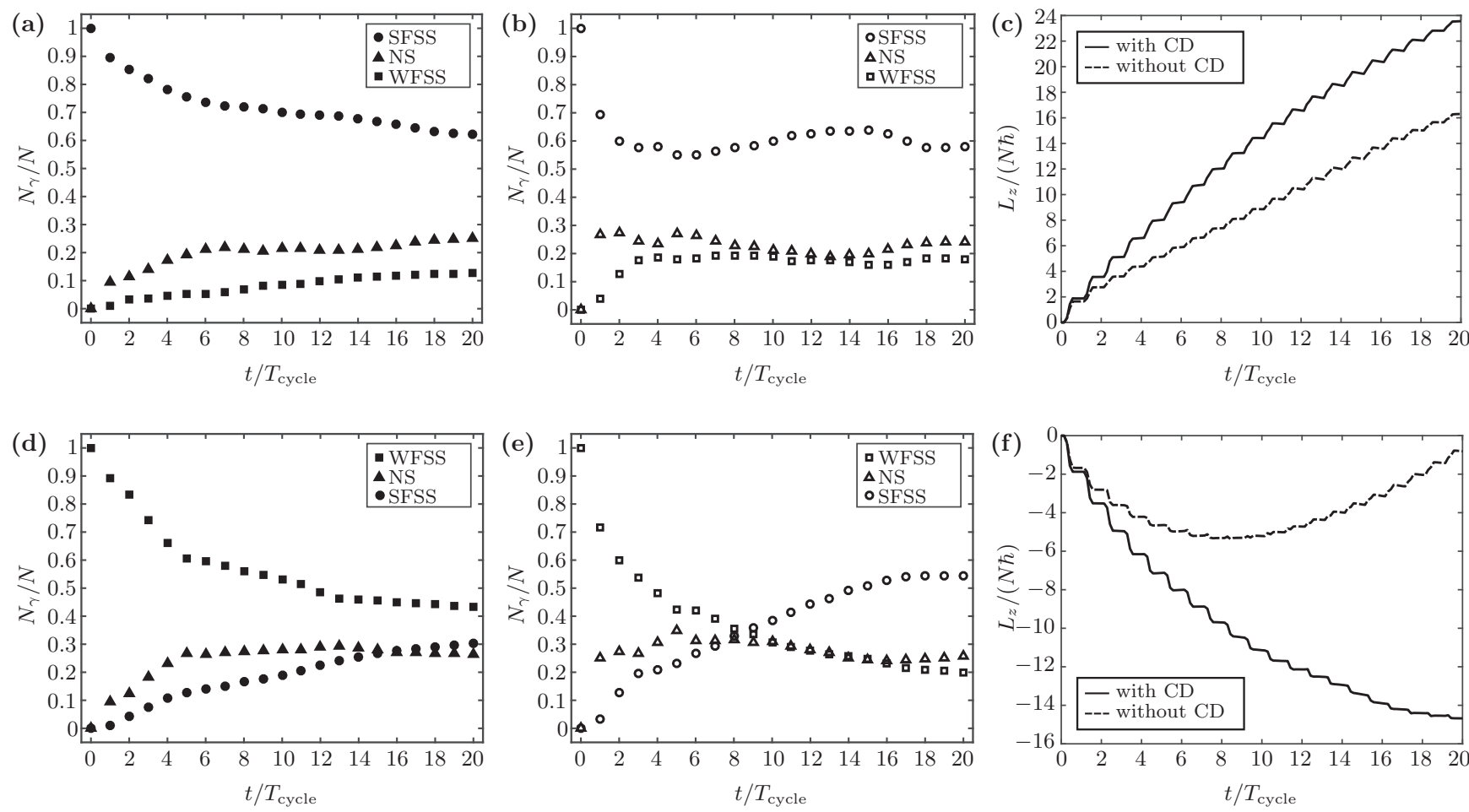

FIG. 4. (a),(b),(d),(e) Particle number $N_{\gamma}$ for the Zeeman eigenstate $\gamma \in\{$ WFSS,NS,SFSS $\}$ as a function of time (a),(d) with and (b),(e) without the CD field. (c),(f) Orbital angular momentum along $z$ as a function of time. The initial state is (a)-(c) SFSS and (d)-(f) WFSS.

field is used or not. Here, also, the effect of the CD field is negligibly small because $\dot{\alpha}(t)$ is small.

\section{Effect of condensate aspect ratio on the vortex creation fidelity}

In the derivation of the CD field of Eq. (6) we have set $z=0$. Hence the CD field is most beneficial for highly oblate rather than spherical or prolate BECs. In Fig. 3, we study the effect of the aspect ratio of the BEC cloud on the vortex creation fidelity by varying the aspect ratio of the trap frequencies $\omega_{z} / \omega_{\rho}$.

We find that, in general, the vortex creation process is more precise with more oblate condensates. This is the case regardless whether we choose to include the $\mathrm{CD}$ field in the creation process or not. Without the CD field, however, the fidelity saturates well below unity if $\omega_{\rho}$ is kept constant. In contrast, if $\omega_{\rho}$ is varied, the effective condensate width changes. Due to the increase in the effective width, also the region in which the vortex creation is nearly adiabatic is increased, providing higher vortex creation fidelities.

\section{VORTEX PUMPING WITH COUNTERDIABATIC QUANTUM CONTROL}

Let us apply the CD control to vortex pumping, in which we cyclically increase the angular momentum of the vortex hosted in the BEC. Here, we choose $\omega_{z} / \omega_{\rho}=5$ with $\omega_{z}=2 \pi \times 124 \mathrm{~Hz}, \rho_{0}=13.4 \mu \mathrm{m}$, and employ the optical plug to ensure that, with the CD field, roughly $90 \%$ of the atoms will remain in the initial state after the first vortex pumping cycle. Furthermore, the optical plug serves to prevent the created multiquantum vortex from splitting into multiple single-quantum vortices [35]. Here, we consider vortex pumping up to 20 cycles. Each cycle consists of the following sequential steps:

(I) The quadrupole field strength $b_{\mathrm{q}}$ is linearly set from zero to $3.675 \mathrm{G} / \mathrm{cm}$ in $T_{1}=0.06 \mathrm{~ms}$, while keeping the bias field $B_{0}$ fixed at $B_{\mathrm{i}}=0.1 \mathrm{G}$.

(II) The bias field $B_{0}$ is ramped to $-B_{\mathrm{i}}$ using the nonlinear ramp with the CD field in $T_{2}=1.16 \mathrm{~ms}$.

(III) The quadrupole field strength $b_{\mathrm{q}}$ is linearly ramped from $3.675 \mathrm{G} / \mathrm{cm}$ to zero in $T_{3}=0.06 \mathrm{~ms}$.

(IV) The bias field $B_{0}$ is linearly rotated to its initial state in $T_{4}=0.51 \mathrm{~ms}$, while keeping its magnitude constant. Here we utilize an additional bias field in the $y$ direction, which is ramped up and down during the field rotation.

Steps I, III, and IV have essentially no effect in the relative populations of the Zeeman eigenstates. Here we have chosen $B_{\mathrm{i}}=0.1 \mathrm{G}$, instead of $B_{\mathrm{i}}=0.5 \mathrm{G}$, to reduce the ramp time by a factor of 5 compared with Fig. 2, while the amount of nonadiabatic excitations remain constant. ${ }^{1}$ We numerically verified that the simulations give equivalent results if the additional bias field is applied along $x$ instead of $y$ in step IV. The total duration of one cycle in this scheme is only $T_{\text {cycle }}=1.79 \mathrm{~ms}$. This is significantly faster than vortex pumping relying on standard adiabatic dynamics [28-31,33].

The particle numbers for the Zeeman eigenstates and the $z$ components of the condensate orbital angular momentum, $L_{z}=-i \hbar \int d \boldsymbol{r} \Psi^{\dagger}\left(x \partial_{y}-y \partial_{x}\right) \Psi$, at different stages of the

\footnotetext{
${ }^{1}$ We may use $B_{\mathrm{i}}=0.1 \mathrm{G}$ because, for the chosen ramp time, $\alpha(t)$ is negligibly small, and consequently $\Psi^{\prime}(\boldsymbol{r}, t) \approx \Psi(\boldsymbol{r}, t)$ in the beginning and at the end of step II.
} 
vortex pumping process are shown in Fig. 4. In the beginning of the simulation and at the end of step IV, the particle numbers for each cycle can be conveniently evaluated as $N_{\mathrm{WFSS}}=$ $\int d \boldsymbol{r}\left|\psi_{-1}\right|^{2}, N_{\mathrm{NS}}=\int d \boldsymbol{r}\left|\psi_{0}\right|^{2}$, and $N_{\mathrm{SFSS}}=\int d \boldsymbol{r}\left|\psi_{+1}\right|^{2}$. After 20 cycles, the CD protocol yields an orbital angular momentum of approximately $-14 N \hbar$ and $23 N \hbar$ with the initial conditions corresponding to the WFSS and SFSS, respectively. This is a clear improvement compared with the case without the CD protocol, for which approximately $-6 N \hbar$ is reached at the ninth cycle and $16 N \hbar$ is reached

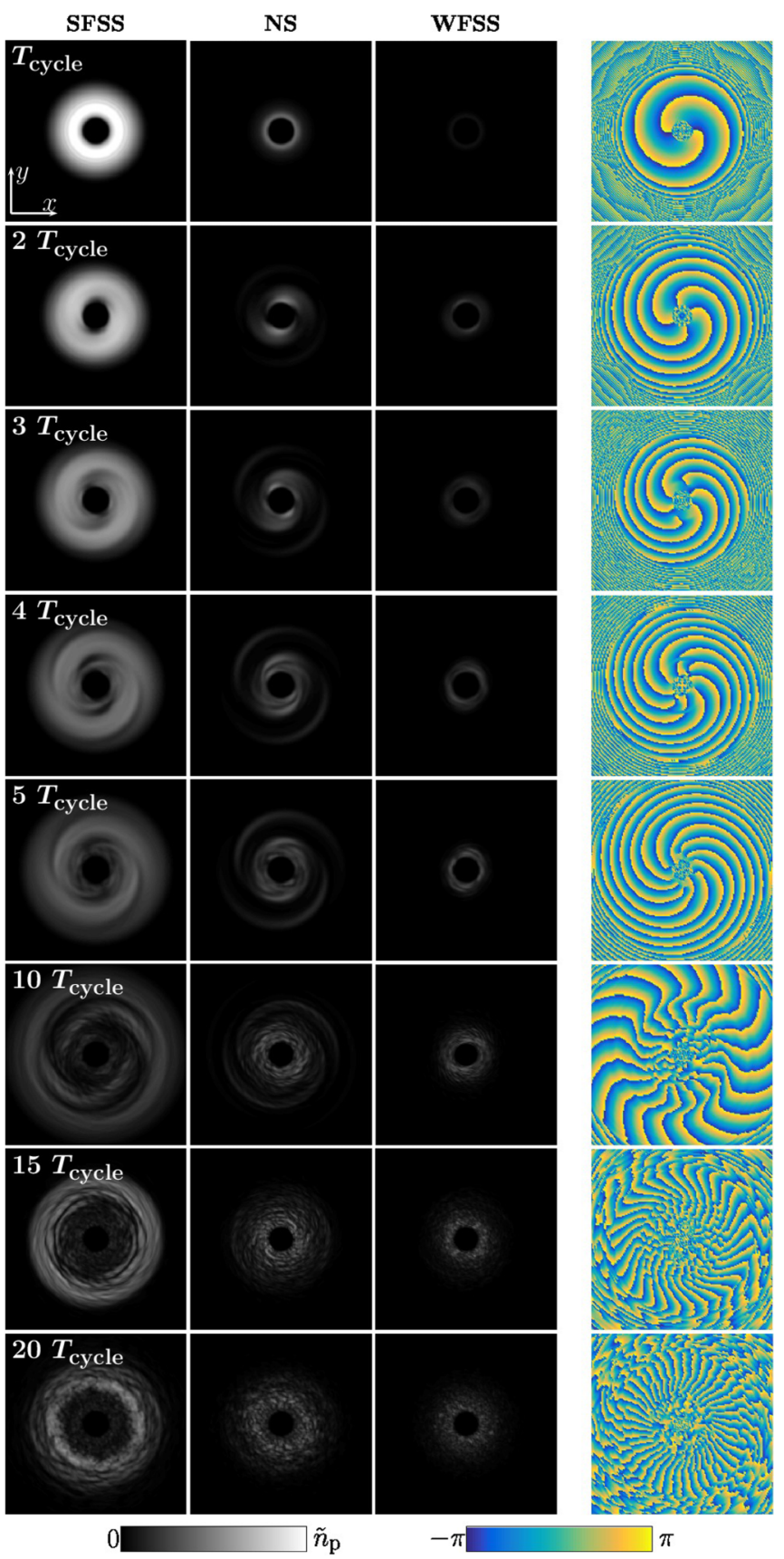

FIG. 5. Particle densities of the SFSS, NS, and WFSS integrated along the $z$ axis, corresponding to Fig. 4(f). The field of view in each panel is $30 \times 30 \mu \mathrm{m}^{2}$ and the maximum particle density is $\tilde{n}_{\mathrm{p}}=1.52 \times 10^{11} \mathrm{~cm}^{-2}$. The rightmost column shows the phase of SFSS in the $z=0$ plane. at the end of the twentieth cycle, for the initial conditions corresponding to the WFSS and SFSS, respectively. In an ideal case, a 20-cycle vortex pumping process yields orbital angular momentum of $\pm 40 \mathrm{~N} \hbar$, since each cycle provides an additional $\pm 2 N \hbar$. This value is not achieved due to the nonadiabatic transitions occurring at every cycle between the different Zeeman eigenstates. Thus the evolving state is a combination of WFSS, NS, and SFSS.

For WFSS as an initial state, in the case without the CD field, the orbital angular momentum starts increasing roughly at the ninth cycle when SFSS becomes the dominant state. For SFSS as an initial state, it remains as the dominant state throughout the 20-cycle process. The strong-field-seeking state yields higher fidelity in vortex pumping because in comparison to the WFSS, the condensate accumulates further away from the field zero into the region where the spin rotations caused by the external magnetic field are more adiabatic.

The order parameters at various instants of time in the course of vortex pumping are shown in Fig. 5. Here, the initial state is SFSS and the CD protocol is applied. The phase information reveals the precise accumulation of the winding number during the pumping process although the angular momentum does not exactly increase by $2 N \hbar$ per cycle. The spiraling phase pattern is attributed to the additional breathing of the condensate during the vortex creation process.

\section{CONCLUSIONS}

We have numerically studied topological vortex imprinting and vortex pumping in spinor BECs of ${ }^{87} \mathrm{Rb}$ atoms aided by counterdiabatic quantum control. The employed CD control can be achieved with a single set of quadrupole field coils and the simulation parameters are chosen to be experimentally feasible. We demonstrate that the CD field can be used to reduce the atom loss in the topological vortex imprinting process also in the case of an optical plug. The highest fidelity in the nonadiabatic regime in the vortex creation process is achieved in our simulations with nonlinear ramps employing both the optical plug and the CD scheme. We also find that the more oblate the condensate, the higher the vortex creation fidelity. Importantly, we show that the CD control and the optical plug can be used to accelerate the vortex pumping process in comparison to the standard adiabatic protocol. This speedup leads to the highest angular momentum per particle reported to date for the vortex pump.

The experimental realization of the vortex pump remains a milestone to be achieved in the studies of topological defects in spinor BECs. Our results show that the requirement of adiabaticity in conventional vortex pumping can be relaxed by employing the $\mathrm{CD}$ scheme. Faster pumping also acts to prevent the splitting of vortices with large winding number during the pumping process.

\section{ACKNOWLEDGMENTS}

We thank David Hall for insightful discussions and Pekko Kuopanportti for valuable comments on the manuscript. This research has been supported by the Academy of Finland through its Centres of Excellence Program (Grants No. 251748 and No. 284621), Japan Society for the Promotion of Science 
(JSPS) Grant-in-Aid for Young Scientists (B) (No. 24740254), JSPS Postdoctoral Fellowships for Research Abroad, and JSPS Grants-in-Aid for Scientific Research (Grant No. 26400422).
In addition, CSC-IT Center for Science Ltd. (Project No. ay2090) and Aalto Science-IT Project are acknowledged for computational resources.
[1] M. H. Anderson, J. R. Ensher, M. R. Matthews, C. E. Wieman, and E. A. Cornell, Science 269, 198 (1995).

[2] K. B. Davis, M.-O. Mewes, M. R. Andrews, N. J. van Druten, D. S. Durfee, D. M. Kurn, and W. Ketterle, Phys. Rev. Lett. 75, 3969 (1995).

[3] A. L. Fetter, Rev. Mod. Phys. 81, 647 (2009).

[4] N. D. Mermin, Rev. Mod. Phys. 51, 591 (1979).

[5] M. Nakahara, Geometry, Topology and Physics, 2nd ed. (Taylor \& Francis, Boca Raton, FL, 2003).

[6] M. Nakahara, T. Isohima, K. Machid, S.-i. Ogawa, and T. Ohmi, Physica B 284-288, 17 (2000).

[7] T. Isoshima, M. Nakahara, T. Ohmi, and K. Machida, Phys. Rev. A 61, 063610 (2000).

[8] S.-i. Ogawa, M. Möttönen, M. Nakahara, T. Ohmi, and H. Shimada, Phys. Rev. A 66, 013617 (2002).

[9] M. Möttönen, N. Matsumoto, M. Nakahara, and T. Ohmi, J. Phys.: Condens. Matter 14, 13481 (2002).

[10] Y. Kawaguchi, M. Nakahara, and T. Ohmi, Phys. Rev. A 70, 043605 (2004).

[11] Y. Kawaguchi, M. Nakahara, and T. Ohmi, J. Low Temp. Phys. 138, 699 (2005).

[12] For a review, see V. Pietilä, M. Möttönen, and M. Nakahara, in Electromagnetic, Magnetostatic, and Exchange-Interaction Vortices in Confined Magnetic Structures, edited by E. O. Kamenetskii (Transworld Research Network, Trivandrum, Kerala, India, 2008), p. 297.

[13] A. E. Leanhardt, A. Görlitz, A. P. Chikkatur, D. Kielpinski, Y. Shin, D. E. Pritchard, and W. Ketterle, Phys. Rev. Lett. 89, 190403 (2002).

[14] A. E. Leanhardt, Y. Shin, D. Kielpinski, D. E. Pritchard, and W. Ketterle, Phys. Rev. Lett. 90, 140403 (2003).

[15] Y. Shin, M. Saba, M. Vengalattore, T. A. Pasquini, C. Sanner, A. E. Leanhardt, M. Prentiss, D. E. Pritchard, and W. Ketterle, Phys. Rev. Lett. 93, 160406 (2004).

[16] M. Kumakura, T. Hirotani, M. Okano, Y. Takahashi, and T. Yabuzaki, Phys. Rev. A 73, 063605 (2006).

[17] T. Isoshima, M. Okano, H. Yasuda, K. Kasa, J. A. M. Huhtamäki, M. Kumakura, and Y. Takahashi, Phys. Rev. Lett. 99, 200403 (2007).

[18] T. Kuwamoto, H. Usuda, S. Tojo, and T. Hirano, J. Phys. Soc. Jpn. 79, 034004 (2010).

[19] H. Shibayama, Y. Yasaku, and T. Kuwamoto, J. Phys. B 44, 075302 (2011).

[20] V. Pietilä and M. Möttönen, Phys. Rev. Lett. 103, 030401 (2009).

[21] M. W. Ray, E. Ruokokoski, S. Kandel, M. Möttönen, and D. S. Hall, Nature (London) 505, 657 (2014).

[22] M. R. Matthews, B. P. Anderson, P. C. Haljan, D. S. Hall, C. E. Wieman, and E. A. Cornell, Phys. Rev. Lett. 83, 2498 (1999).

[23] K. W. Madison, F. Chevy, W. Wohlleben, and J. Dalibard, Phys. Rev. Lett. 84, 806 (2000).

[24] S. Inouye, S. Gupta, T. Rosenband, A. P. Chikkatur, A. Görlitz, T. L. Gustavson, A. E. Leanhardt, D. E. Pritchard, and W. Ketterle, Phys. Rev. Lett. 87, 080402 (2001).
[25] E. Hodby, G. Hechenblaikner, S. A. Hopkins, O. M. Maragò, and C. J. Foot, Phys. Rev. Lett. 88, 010405 (2001).

[26] M. F. Andersen, C. Ryu, P. Cladé, V. Natarajan, A. Vaziri, K. Helmerson, and W. D. Phillips, Phys. Rev. Lett. 97, 170406 (2006).

[27] D. R. Scherer, C. N. Weiler, T. W. Neely, and B. P. Anderson, Phys. Rev. Lett. 98, 110402 (2007).

[28] M. Möttönen, V. Pietilä, and S. M. M. Virtanen, Phys. Rev. Lett. 99, 250406 (2007).

[29] Z. F. Xu, P. Zhang, C. Raman, and L. You, Phys. Rev. A 78, 043606 (2008).

[30] Z. F. Xu, P. Zhang, R. Lü, and L. You, Phys. Rev. A 81, 053619 (2010).

[31] P. Kuopanportti, B. P. Anderson, and M. Möttönen, Phys. Rev. A 87, 033623 (2013).

[32] Y. Kawaguchi and T. Ohmi, Phys. Rev. A 70, 043610 (2004).

[33] P. Kuopanportti and M. Möttönen, J. Low Temp. Phys. 161, 561 (2010).

[34] P. Kuopanportti, E. Lundh, J. A. M. Huhtamäki, V. Pietilä, and M. Möttönen, Phys. Rev. A 81, 023603 (2010).

[35] P. Kuopanportti and M. Möttönen, Phys. Rev. A 81, 033627 (2010).

[36] M. Demirplak and S. A. Rice, J. Phys. Chem. A 107, 9937 (2003)

[37] M. Berry, J. Phys. A 42, 365303 (2009).

[38] E. Torrontegui, S. Ibáñez, S. Martínez-Garaot, M. Modugno, A. del Campo, D. Guéry-Odelin, A. Ruschhaupt, X. Chen, and J. G. Muga, Adv. At. Mol. Opt. Phys. 62, 117 (2013).

[39] S. Masuda and S. A. Rice, Controlling Quantum Dynamics with Assisted Adiabatic Processes (John Wiley \& Sons, Hoboken, NJ, 2016), Vol. 159, Chap. 3.

[40] S. Masuda and S. A. Rice, J. Phys. Chem. C 119, 14513 (2014).

[41] X. Chen, I. Lizuain, A. Ruschhaupt, D. Guéry-Odelin, and J. G. Muga, Phys. Rev. Lett. 105, 123003 (2010).

[42] A. del Campo, M. M. Rams, and W. H. Zurek, Phys. Rev. Lett. 109, 115703 (2012).

[43] K. Takahashi, Phys. Rev. E 87, 062117 (2013).

[44] M. G. Bason, M. Viteau, N. Malossi, P. Huillery, E. Arimondo, D. Ciampini, R. Fazio, V. Giovannetti, R. Mannella, and O. Morsch, Nat. Phys. 8, 147 (2011).

[45] A. del Campo, Phys. Rev. Lett. 111, 100502 (2013).

[46] J. Zhang, J. H. Shim, I. Niemeyer, T. Taniguchi, T. Teraji, H. Abe, S. Onoda, T. Yamamoto, T. Ohshima, J. Isoya, and D. Suter, Phys. Rev. Lett. 110, 240501 (2013).

[47] S. Masuda, U. Güngördü, X. Chen, T. Ohmi, and M. Nakahara, Phys. Rev. A 93, 013626 (2016).

[48] M. W. Ray, E. Ruokokoski, K. Tiurev, M. Möttönen, and D. S. Hall, Science 348, 544 (2015).

[49] T. Ohmi and K. Machida, J. Phys. Soc. Jpn. 67, 1822 (1998).

[50] T.-L. Ho, Phys. Rev. Lett. 81, 742 (1998).

[51] E. G. M. van Kempen, S. J. J. M. F. Kokkelmans, D. J. Heinzen, and B. J. Verhaar, Phys. Rev. Lett. 88, 093201 (2002).

[52] M. W. Berry, Proc. R. Soc. London A 392, 45 (1984). 